\title{
The Intramolecular Loss of Fluorescence by Lysine Derivatized with Naphthalenedialdehyde
}

\author{
STEVEN A. SOPER, STEVE CHAMBERLIN, CAREY K. JOHNSON, and \\ THEODORE KUWANA* \\ Center for Bioanalytical Research and the Department of Chemistry, the University of Kansas, Lawrence, Kansas 66045
}

\begin{abstract}
Derivatization of primary amines such as amino acids and peptides with naphthalenedialdehyde (NDA) in the presence of cyanide ion yields cyanobenzo[f]-isoindole (CBI) adducts that are highly fluorescent. However, the fluorescence is seriously quenched with amines that possess more than one primary amine site, as is the case with lysine. Although it was found that the adsorption of $\mathrm{CBI}_{2}$-lysine on a solid substrate restored the fluorescence, the reason for the solution quenching, with respect to results for mono-derivatized amines, was investigated. The experiments to probe the quenching were based on the assumption that the mechanism responsible for quenching involved a charge-transfer (CT) excited state. Thus, it was found that the solvent properties of viscosity and polarity affected the lifetime and quantum yield of fluorescence in a manner consistent with the proposed mechanism.

Index Headings: Fluorescence; Analytical methods.
\end{abstract}

\section{INTRODUCTION}

Tagging of biological substances by derivatization to yield highly fluorescent products is an important strategy to achieve high-sensitivity liquid chromatographic analysis. For example, naphthalenedialdehyde (NDA) in the presence of cyanide ion is an excellent reagent for the derivatization of primary amines, such as amino acids and peptides, to give the fluorescent adduct, the cyanobenzo[f]isoindole (CBI). ${ }^{1-4}$ These adducts have fluorescence quantum yields approaching unity and exhibit exceptional stability, making possible pre-column derivatization schemes for LC analysis. Thus, high-sensitivity detection in the attomole range has been reported for several different CBI-amines with laser-induced fluorescence detection in HPLC. ${ }^{5-7}$

One limitation of the NDA/CN reagent occurs when the primary amine possesses more than one derivatization site. The product of the derivatization, such as in the case of lysine, is $\mathrm{CBI}_{2}$-lysine, which exhibits a substantially lower fluorescence quantum yield than those observed for the mono-derivatized CBI-amines. ${ }^{8}$ However, the quantum yield increases substantially when $\mathrm{CBI}_{2}$-lysine is adsorbed on a solid TLC substrate, becoming similar to that of the mono-derivatized amine. ${ }^{9}$ For example, in Fig. 1 the fluorescence excitation and emission spectra of $\mathrm{CBI}_{2}$-lysine is compared with those of CBI-alanine when they are both adsorbed on a TLC matrix. The fluorescence emission intensities are similar in magnitude in the adsorbed case; whereas in solution, a 20 -fold difference exists, with $\mathrm{CBI}_{2}$-lysine having a much lower quantum yield $\left(\phi_{f}=0.02\right)$.

The above observations stimulated the present investigation to find the reason for the loss of fluorescence for

Received 12 December 1989.

* Author to whom correspondence should be sent. a multi-derivatized amine such as lysine. A possible mechanism to explain the solution quenching of this $\mathrm{CBI}_{2}-$ lysine is the formation of a charge-transfer (CT) state, which competes favorably with the fluorescence de-excitation from the first, locally excited (LE), state. Such a CT state would require a molecular and conformational structure that is not possible with mono-derivatized CBI adducts. These CT or excimer states have been well documented in the literature. For example, naphthylalkylamines exhibit intramolecular exciplex formation, in which a decrease in the temperature of the solvent (higher viscosity) results in an increase in the fluorescence from the monomeric naphthalene and a corresponding decrease in the exciplex emission. ${ }^{10}$ The viscosity dependence for excimer fluorescence is due to the fact that the electron donor and acceptor must attain a "sandwich" conformation during the lifetime of the excited state. ${ }^{11-13}$ An increase in the viscosity serves to lower the rate at which this conformation can be attained.

Charge transfer complexes have been shown to be stabilized by increasing the polarity of the solvent. Amiri ${ }^{14}$ demonstrated that the quantum efficiency and decay time of the intramolecular charge transfer complex of 4-dicyanomethylene-1,2,3,4-tetrahydromethylquinoline increased as the polarity of the solvent was increased. $p$-Dimethylamino-benzonitrile (DMABN) exhibited dual fluorescence only in polar solvents with the absence of the CT fluorescence band in nonpolar solvents. ${ }^{15}$ Eisenthal and co-workers ${ }^{16,17}$ determined that the rate of CT formation was dependent on the polarity of the solvent, the rate increasing with more polar solvents. In addition, the CT fluorescence band of DMABN was found to be absent in very rigid matrices due to the inability of the molecule to undergo the necessary conformational change associated with CT formation. ${ }^{18}$

The fluorescence properties of $\mathrm{CBI}_{2}$-lysine are compared to those of CBI-alanine in this study. Their spectral properties and fluorescence yields have been previously documented. ${ }^{3}$ CBI-alanine possesses only one derivatization site, while $\mathrm{CBI}_{2}$-lysine has two possible sites. The proposed energy diagram showing energy states for $\mathrm{CBI}_{2}$-lysine is presented in Fig. 2. There are three manifolds for depopulating the $\mathrm{LE}$ state of $\mathrm{CBI}_{2}$-lysine. Decay to the ground state can occur via fluorescence $\left(k_{A F}\right)$ or through a nonradiative process at a rate designated as $k_{A N}$. In addition, depletion of population from the LE state can occur through formation of a proposed CT state $\left(k_{A B}\right)$. The rate or efficiency of CT formation should be affected by the solvent viscosity, if formation of the CT state requires a conformational change during the lifetime of the excited state. Solvent polarity also affects the rate of CT formation, since an increase in the dipole 

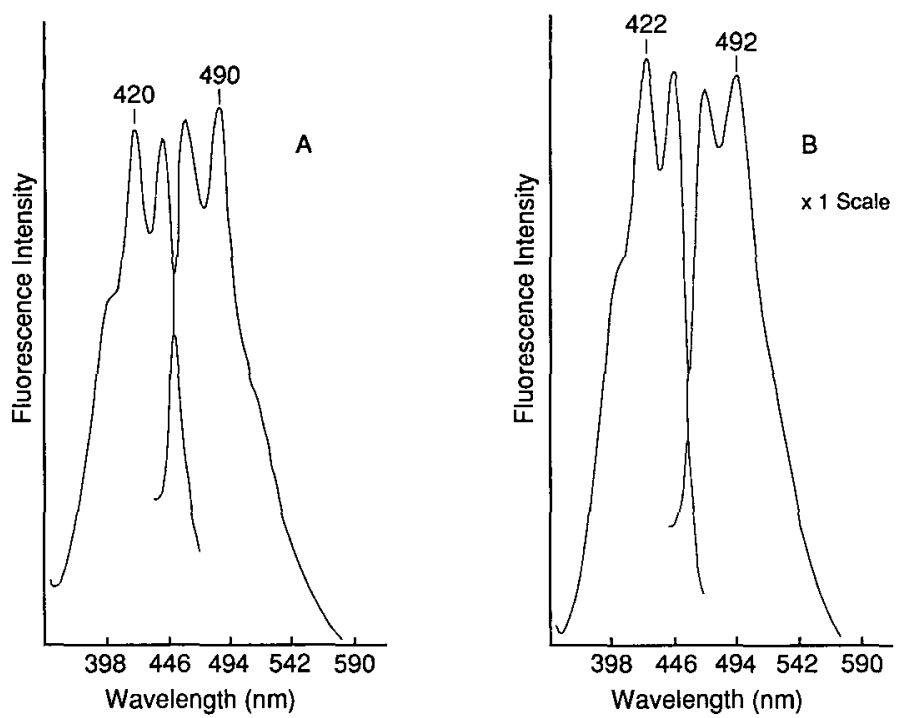

FIG. 1. The fluorescence excitation and emission spectra of $1 \times 10^{-5}$ $\mathrm{M}$ of $\mathrm{CBI}$-alanine and $\mathrm{CBI}_{2}$-lysine deposited onto an aluminum oxide TLC surface. The emission spectra were obtained at an excitation wavelength of $420 \mathrm{~nm}$.

moment of the CT state would be favored in more polar solvents. This rate can be indirectly evaluated as a function of solvent viscosity and polarity by monitoring how these solvent parameters affect the fluorescence quantum efficiency and lifetime of the LE state.

The energy diagram for CBI-alanine is also shown in Fig. 2. The formation of a CT state cannot occur in this derivative due to the lack of a second isoindole ring (electron acceptor or donor). Thus, only fluorescence and nonradiative processes serve to depopulate the excited state. The fluorescence quantum efficiency and lifetime of CBIalanine, measured and compared with those obtained for the $\mathrm{CBI}_{2}$-lysine system in solvents of various viscosities and polarities, are discussed herein.

\section{EXPERIMENTAL}

Reagents and Instrumentation. Alanine, lysine, and sodium cyanide were obtained from Sigma Co. (St. Louis, MO) and used without further purification. Naphthalenedialdehyde (NDA) was synthesized and purified according to published procedures. ${ }^{1}$ All solvents were obtained from Aldrich Co. (Milwaukee, WI) and used as received. Fluorescence spectra were acquired on a Perkin-Elmer LS-5 fluorescence spectrophotometer (Oak Brook, IL). Fluorescence quantum efficiencies were calculated relative to CBI-alanine in $100 \% \mathrm{H}_{2} \mathrm{O}$ as a standard, since its quantum efficiency has been reported in the literature. ${ }^{3}$ Fluorescence lifetimes were measured by the phase/modulation technique on an SLM Aminco 4800 spectrofluorometer (Urbana, IL) with modulation frequencies of 6,18 , and $30 \mathrm{MHz}$. This instrument allowed the measurement of lifetimes as short as $1 \mathrm{~ns}$. Constant temperatures were maintained by circulating water/ethylene glycol from a refrigerated circulating bath (Neslab, Newington, $\mathrm{NH}$ ) through a jacketed fluorescence cuvette (Uvonic Instrument Inc., Plainview, NY). All solutions were purged with $\mathrm{N}_{2}$ prior to spectral acquisition. Molecular modeling was performed on a Digital Equipment

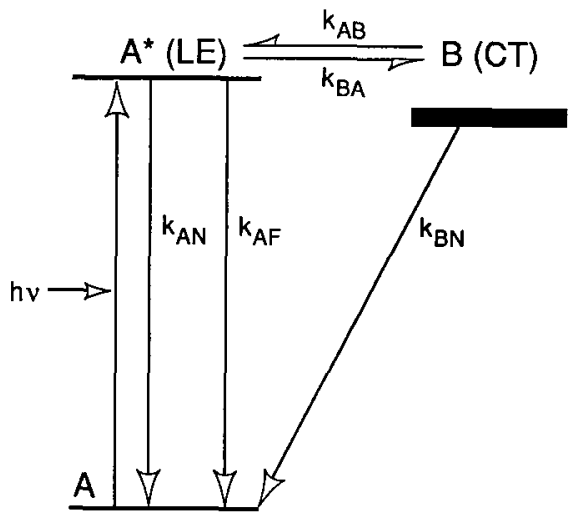

(1)

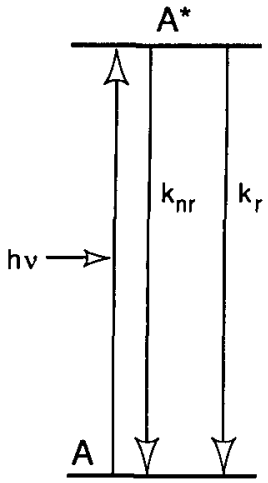

(2)
FIG. 2. The proposed model for the intramolecular fluorescence loss of $\mathrm{CBI}_{2}$-lysine. Energy level diagram of (1) $\mathrm{CBI}_{2}$-lysine and (2) CBIalanine.

MicroVAX II computer (Maymard, MA) utilizing MacroModel version II software (Columbia University).

Synthesis of the CBI-amines. To a two-necked roundbottom flask, $25 \mathrm{~mL}$ of methanol, $147.2 \mathrm{mg}$ of NDA $(0.8$ $\mathrm{mmol})$, and $40 \mathrm{mg}$ of $\mathrm{NaCN}(0.8 \mathrm{mmol})$ were added and mixed. One neck of the flask was stoppered with a septum, and a stream of argon was introduced through the other. The reaction flask was immersed into an acetone/ dry-ice bath so that low temperatures could be maintained. The amine was dissolved in $1 \mathrm{~mL}$ of $\mathrm{H}_{2} \mathrm{O}$ and introduced into the reaction flask via a syringe through the septum. The mixture was allowed to react for $\sim 1 \mathrm{~h}$ with constant stirring. After the reaction was completed, the solvent was removed under reduced pressure with a residue remaining that was green (CBI-alanine) or yellow $\left(\mathrm{CBI}_{2}\right.$-lysine). The residue was dissolved in a small amount of methanol and then recrystallized with ether. The isolated crystals were filtered and dried under a stream of argon. The purity and identification of the derivatives were confirmed by proton and C-13 NMR and IR spectroscopy.

\section{RESULTS}

The electronic absorption spectra of CBI-alanine and $\mathrm{CBI}_{2}$-lysine are very similar, with both showing absorption maxima at $\sim 420$ and $440 \mathrm{~nm}$. The positions of these maxima were unaffected by changes in solvent polarity or viscosity. Measurement of the molar absorptivities for both adducts indicated a twofold increase for $\mathrm{CBI}_{2}$-lysine, in comparison to CBI-alanine. The fluorescence emission spectra of these two derivatives displayed a maximum emission at $\sim 485 \mathrm{~nm}$, with the position of this maximum being insensitive to changes in the properties of the solvent. Careful inspection of the fluorescence emission spectra of $\mathrm{CBI}_{2}$-lysine failed to show the presence of any band red-shifted from the fluorescence band at $485 \mathrm{~nm}$. The only observed spectral difference between the fluorescence emission of $\mathrm{CBI}$-alanine and $\mathrm{CBI}_{2}$-lysine was the intensity of the emission, the former being more intense. The similarities in the wavelength maxima of the absorption and emission spectra of both adducts indicate that the ground and fluorescent electronic states of $\mathrm{CBI}_{2}$-lysine are very similar to those of CBI-alanine. 
TABLE I. The viscosities and $E_{t}(30)$ values of the solvents used in this investigation. Temperatures resulting in the displayed viscosities are given in parentheses. ${ }^{a}$

\begin{tabular}{lcc}
\hline \multicolumn{1}{c}{ Solvent } & $\eta(\mathrm{cp})$ & $E_{t}(30)(\mathrm{kcal} / \mathrm{mole})^{\mathrm{b}}$ \\
\hline Glycerol (25) & 954 & 57.0 \\
Water (20) & 1.004 & 63.1 \\
Butanol (20) & 2.948 & 50.2 \\
Butanol (40) & 1.77 & \\
Hexadecane (20) & 3.34 & 30.8 \\
Methanol (20) & 0.597 & 55.5 \\
Chloroform (20) & 0.58 & 39.1 \\
Ethanol (0) & 1.77 & 51.9 \\
Propanol (30) & 1.77 & 50.7 \\
\hline
\end{tabular}

a Temperatures are in degrees Celsius.

b Taken from Ref. 20.

The value of the radiative rate constant, $k_{r}$, of the $\mathrm{LE}$ state for CBI-alanine and $\mathrm{CBI}_{2}$-lysine can be calculated from the integrated absorption and fluorescence spectra according to Eq. 1: ${ }^{19}$

$$
k_{r}=2.88 \times 10^{9} \eta^{2}\left\langle\nu_{f}^{3}\right\rangle_{A V}{ }^{-1} \int \epsilon \operatorname{dln} \nu
$$

where $\eta$ is the refractive index of the given solvent, $\left\langle\nu_{f}^{-3}\right\rangle_{A V}{ }^{-1}$ is obtained experimentally from the integration of the fluorescence emission spectrum and the integration of the same spectrum multiplied by the cubed intensity value at each frequency. $\int \epsilon \operatorname{dln} \nu$ is the integrated extinction coefficient, with $k_{r}$ being the radiative rate constant ( $k_{A F}$ in Fig. 2, diagram 1). The value of $k_{r}$ calculated from Eq. 1 for CBI-alanine in 90:10 MeOH: $\mathrm{H}_{2} \mathrm{O}$ is $0.044 \mathrm{~ns}^{-1}$. A similar value of $0.039 \mathrm{~ns}^{-1}$ is obtained for $\mathrm{CBI}_{2}$-lysine. The radiative rate constant for CBIalanine was also calculated through the use of Eqs. 2 and

TABLE II. The fluorescence lifetime $\left(\tau_{f}\right)$ and quantum efficiencies $\left(\phi_{f}\right)$ for CBI-alanine and $\mathrm{CBI}_{2}$-lysine in butanol/hexadecane binary mixtures. The radiative $\left(k_{r}\right)$ and nonradiative $\left(k_{n r}\right)$ rate constants for CBI-alanine are also presented (calculated with Eqs. 2 and 3).a

\begin{tabular}{ccccc}
\hline $\begin{array}{c}\text { CBI alanine } \\
\% \text { Butanol }\end{array}$ & $\tau_{f}(\mathrm{~ns})$ & $\phi_{f}$ & $k_{r}\left(\mathrm{~ns}^{-1}\right)$ & $k_{n r}\left(\mathrm{~ns}^{-1}\right)$ \\
\hline 90 & 13.3 & $\begin{array}{c}0.62 \\
( \pm 0.7)\end{array}$ & $\begin{array}{c}( \pm 0.03) \\
0.047\end{array}$ & 0.027 \\
& 13.0 & 0.63 & 0.045 & 0.027 \\
80 & $( \pm 0.6)$ & $( \pm 0.03)$ & & \\
70 & 14.3 & 0.62 & 0.043 & 0.027 \\
& $( \pm 0.7)$ & $( \pm 0.02)$ & & \\
60 & 14.1 & 0.64 & 0.046 & 0.026 \\
& $( \pm 0.7)$ & $( \pm 0.03)$ & & \\
50 & 13.6 & 0.65 & 0.048 & 0.026 \\
& $( \pm 0.6)$ & $( \pm 0.02)$ & & \\
\hline
\end{tabular}

\begin{tabular}{cccc}
$\begin{array}{c}\text { CBI } \\
\text { \% lysine }\end{array}$ & & \\
$\%$ Butanol & $\tau_{f}(\mathrm{~ns})$ & $\phi_{f}$ & $\phi_{f} / \tau_{f}$ \\
\hline 90 & 3.1 & 0.10 & 0.032 \\
& $( \pm 0.3)$ & $( \pm 0.01)$ & \\
80 & 3.9 & 0.12 & 0.031 \\
& $( \pm 0.3)$ & $( \pm 0.01)$ & \\
70 & 5.7 & 0.13 & 0.023 \\
& $( \pm 0.2)$ & $( \pm 0.01)$ & \\
60 & 7.4 & 0.16 & 0.022 \\
& $( \pm 0.3)$ & $( \pm 0.01)$ & \\
50 & 9.0 & 0.19 & 0.021 \\
& $( \pm 0.4)$ & $( \pm 0.01)$ & \\
\hline
\end{tabular}

a The standard deviations are given in parentheses.
TABLE III. The fluorescence quantum efficiencies $\left(\phi_{f}\right)$ and lifetimes $\left(\tau_{f}\right)$ of CBI-alanine and $\mathrm{CBI}_{2}$-lysine in methanol/chloroform binary mixtures. The radiative $\left(k_{r}\right)$ and nonradiative $\left(k_{n r}\right)$ rate constants for CBIalanine are also presented."

\begin{tabular}{ccccc}
\hline $\begin{array}{l}\text { CBI-alanine } \\
\% \text { Methanol }\end{array}$ & $\tau_{f}(\mathrm{~ns})$ & $\phi_{f}$ & $k_{r}\left(\mathrm{~ns}^{-1}\right)$ & $k_{n r}\left(\mathrm{~ns}^{-1}\right)$ \\
\hline 100 & 12.2 & 0.70 & 0.055 & 0.025 \\
& $( \pm 0.6)$ & $( \pm 0.03)$ & & \\
80 & 11.2 & 0.68 & 0.061 & 0.029 \\
& $( \pm 0.8)$ & $( \pm 0.03)$ & & \\
60 & 12.1 & 0.67 & 0.055 & 0.027 \\
& $( \pm 0.7)$ & $( \pm 0.02)$ & & \\
40 & 13.1 & 0.71 & 0.054 & 0.022 \\
& $( \pm 0.8)$ & $( \pm 0.04)$ & & \\
20 & 11.9 & 0.66 & 0.055 & 0.028 \\
& $( \pm 0.7)$ & $( \pm 0.03)$ & & \\
\hline
\end{tabular}

\begin{tabular}{cccc}
$\begin{array}{c}\text { CBI } \\
\text { \% Methane }\end{array}$ & & \\
\hline 100 & $\tau_{f}(\mathrm{~ns})$ & $\phi_{f}$ & $\phi_{f} / \tau_{f}$ \\
\hline & 1.3 & 0.030 & 0.023 \\
80 & 2.2 & $( \pm 0.005)$ & \\
& $( \pm 0.3)$ & $( \pm 0.005)$ & 0.022 \\
60 & 2.8 & 0.059 & 0.021 \\
& $( \pm 0.3)$ & $( \pm 0.007)$ & \\
40 & 4.1 & 0.069 & 0.017 \\
& $( \pm 0.4)$ & $( \pm 0.006)$ & \\
20 & 5.3 & 0.078 & 0.015 \\
& $( \pm 0.4)$ & $( \pm 0.007)$ & \\
\hline
\end{tabular}

The standard deviations are given in parentheses.

3 , from the measured fluorescence quantum efficiency $\left(\phi_{f}\right)$ and lifetime $\left(\tau_{f}\right)$ :

$$
\begin{gathered}
\phi_{f}=k_{r} /\left(k_{r}+k_{n r}\right) \\
\tau_{f}=1 /\left(k_{r}+k_{n r}\right)
\end{gathered}
$$

where $k_{n r}$ is the nonradiative rate constant. $k_{r}$ calculated from these relationships resulted in values that agreed favorably with those from the use of Eq. 1 for the CBIalanine system $\left(k_{r}=0.050 \mathrm{~ns}^{-1}\right.$ in $\left.90: 10 \mathrm{MeOH}: \mathrm{H}_{2} \mathrm{O}\right)$.

The influence of the solvent polarity upon the fluorescence emission properties of the CBI-derivatives was investigated in isoviscous solvents in order to minimize viscosity effects. ${ }^{16,17}$ Binary mixtures of solvents whose room-temperature viscosities are equivalent but whose polarities differ dramatically were used. The selected solvents with the values of their viscosities and polarities, as measured by the empirical $E_{t}(30)$ scale, ${ }^{20}$ are listed in Table I.

Butanol and hexadecane have similar viscosities (at $20^{\circ} \mathrm{C}$ ), but differ in their $E_{t}(30)$ values, butanol being the more polar solvent. Therefore, the polarity of the binary mixture can be altered without any significant viscosity change. The fluorescence quantum efficiencies and lifetimes of CBI-alanine and $\mathrm{CBI}_{2}$-lysine, as measured in various butanol/hexadecane compositions, are shown in Table II. The values of $k_{r}$ and $k_{n r}$ for CBI-alanine, as calculated with Eqs. 2 and 3, also appear. The quantum efficiency and lifetime of CBI-alanine, as well as the $k_{r}$ and $k_{n r}$ values, are invariant to changes in the solvent polarity. However, for $\mathrm{CBI}_{2}$-lysine, both $\phi_{f}$ and $\tau_{f}$ increase with a decrease in the solvent polarity.

Methanol and chloroform also have similar viscosities, but differ in their $E_{t}(30)$ values (see Table I). This binary solvent mixture was also investigated for its effect on the 


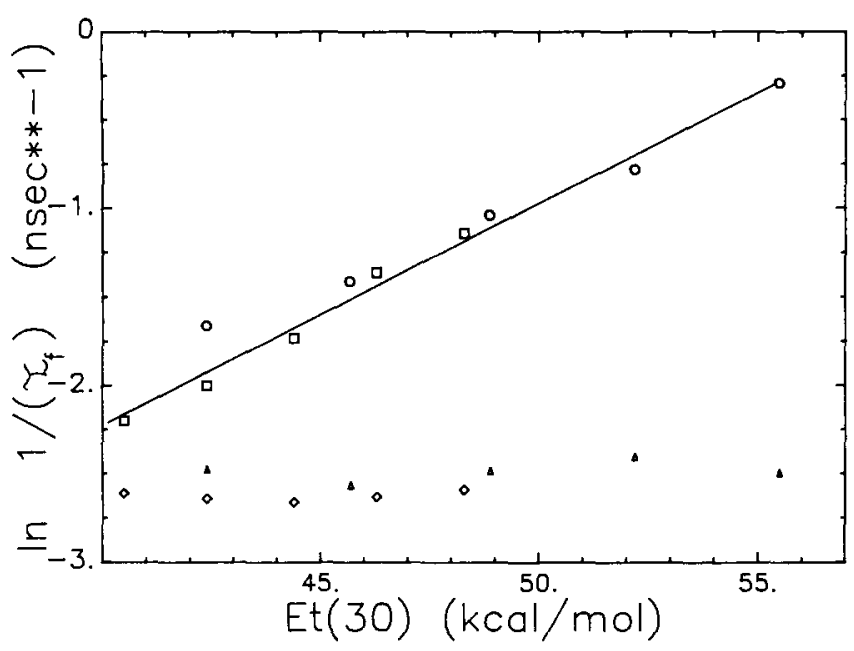

Fig. 3. Values of $\ln \left(1 / \tau_{f}\right)$ versus solvent $E_{t}(30)$ are plotted for $\mathrm{CBI}_{2}$ lysine and CBI-alanine. ( $\square$ ) $\mathrm{CBI}_{2}$-lysine and $(\diamond) \mathrm{CBI}$-alanine in butanol/hexadecane binary mixtures. $(O) \mathrm{CBI}_{2}$-lysine and $(\triangle) \mathrm{CBI}$-alanine in methanol/chloroform binary solvent mixtures.

fluorescence properties of $\mathrm{CBI}$-alanine and $\mathrm{CBI}_{2}$-lysine. The results are tabulated in Table III. Again, the quantum efficiency and lifetime, as well as $k_{r}$ and $k_{n r}$, for CBIalanine, remain constant with respect to changes in the solvent polarity. For $\mathrm{CBI}_{2}$-lysine, both $\phi_{f}$ and $\tau_{f}$ exhibited an increase as the solvent polarity decreased. In Fig. 3, the values of $\ln \left(1 / \tau_{f}\right)$ vs. $E_{t}(30)$ for CBI-alanine and $\mathrm{CBI}_{2^{-}}$ lysine in the binary mixtures of butanol/hexadecane and methanol/chloroform are plotted. A linear dependence of $\ln \left(1 / \tau_{f}\right)$ on the solvent polarity is seen for $\mathrm{CBI}_{2}$-lysine $(r=0.994)$ indicating a dramatic change in the dipole moment for the excited state of $\mathrm{CBI}_{2}$-lysine. In the case of CBI-alanine no such dependency on solvent polarity is observed.

The second method to achieve an isoviscous condition in order to investigate the polarity effects upon the fluorescence properties of $\mathrm{CBI}_{2}$-lysine is to incorporate neat solvents whose $E_{t}(30)$ values differ but whose viscosities may be adjusted to a given value by changing the temperature. The solvents with the temperatures necessary to maintain a viscosity of $1.77 \mathrm{cp}$ are listed in Table I. The results are presented in Table IV for $\mathrm{CBI}_{2}$-lysine. In the alcohol series of ethanol, propanol, and butanol, an increase in the $E_{t}(30)$ value of the solvent resulted in a corresponding decrease in the fluorescence quantum efficiency and lifetime, irrespective of the lower temperature necessary to maintain the isoviscous condition in the more polar solvents. These results are in accordance with the polarity dependence previously obtained. The

TABLE IV. The fluorescence quantum efficiencies $\left(\phi_{f}\right)$ and lifetimes $\left(\tau_{f}\right)$ for $\mathrm{CBI}_{2}$-lysine in neat alcohols at their isoviscous temperatures. ${ }^{\mathrm{a}}$

\begin{tabular}{lcc}
\hline Alcohol & $\tau_{f}(\mathrm{~ns})$ & $\phi_{f}$ \\
\hline Ethanol & 2.4 & 0.046 \\
& $( \pm 0.2)$ & $( \pm 0.006)$ \\
Propanol & 2.8 & 0.060 \\
& $( \pm 0.4)$ & $( \pm 0.007)$ \\
Butanol & 3.0 & 0.070 \\
& $( \pm 0.3)$ & $( \pm 0.008)$ \\
\hline
\end{tabular}

\footnotetext{
${ }^{a}$ The standard deviations are given in parentheses.
}

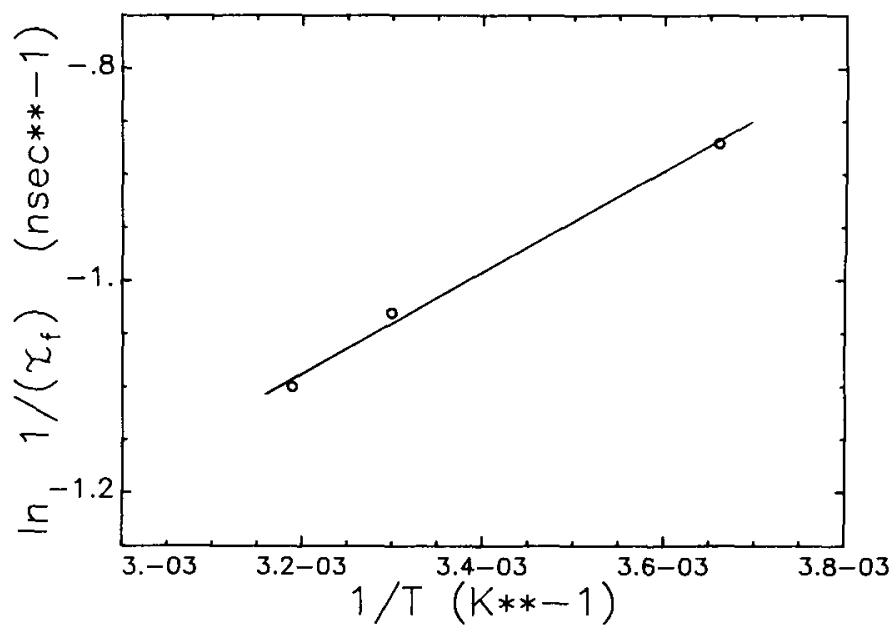

FIg. 4. Plot of $\ln \left(1 / \tau_{f}\right.$ versus $1 / T$ for $\mathrm{CBI}_{2}$-lysine in the alcohol series ethanol, propanol, and butanol.

rate of CT state formation increases in more polar solvents.

An effective activation energy, $E_{\alpha}$, between the LE and CT states of $\mathrm{CBI}_{2}$-lysine can be determined from an Arrhenius-type relationship: ${ }^{16,17}$

$$
\ln 1 / \tau_{f}=\ln \mathrm{B} f(\eta)-E_{a} / R T
$$

where $B$ is the pre-exponential frequency factor and $f(\eta)$ is the viscosity function (which is constant for the isoviscous solvents). A plot of $\ln \left(1 / \tau_{f}\right)$ versus $1 / T$ is shown in Fig. 4. The plot is linear, yielding a negative activation energy, $E_{a}$, of $-0.90 \mathrm{kcal} / \mathrm{mole}$.

The effect of solvent viscosity on the fluorescence of $\mathrm{CBI}$-alanine and $\mathrm{CBI}_{2}$-lysine was evaluated with glycerol/water binary mixtures. Glycerol and water have nearly the same $E_{t}(30)$ values, thereby minimizing polarity effects upon the fluorescence properties. However, their room-temperature viscosities are dramatically different (see Table I). The results are summarized in Table $V$. The fluorescence quantum efficiency and lifetime of CBI-alanine as well as its radiative and nonradiative rate constants were unaffected by changes in the solvent viscosity. For $\mathrm{CBI}_{2}$-lysine, the fluorescence quantum efficiency and lifetime were observed to increase with an increase in the solvent viscosity.

\section{DISCUSSION}

The similarity in the shape and wavelength maxima of the visible absorption spectra of CBI-alanine and $\mathrm{CBI}_{2}{ }^{-}$ lysine indicates that significant electronic interaction between the isoindole rings in the ground-state conformation of $\mathrm{CBI}_{2}$-lysine is absent. This factor, coupled with the molar absorptivity of $\mathrm{CBI}_{2}$-lysine being twice that of the CBI-alanine system, with the maximum in the fluorescence emission occurring at approximately the same wavelength and with the position of this maximum being insensitive to the properties of the solvent, suggests that the observed fluorescence of $\mathrm{CBI}_{2}$-lysine occurs from an excited electronic state nearly identical to that of CBIalanine. We label this state the locally excited (LE) state.

The polarity of the solvent does not affect the energetics of the LE state. However, it does affect the lifetime by influencing the rate of formation of a state which 
competes favorably to diminish the fluorescence quantum yield. The potential energy of such a CT state is expected to be lowered with respect to the LE state as the solvent polarity is increased. This lowering reflects the solvent interaction with the charge-separated CT state. The fluorescence quantum yield and the lifetime of the LE excited state thus correspondingly decrease as the solvent polarity is increased, in accordance with the proposed mechanism.

The radiative rate of the fluorescence lifetime is a function of the five rate constants, as proposed for the levels in Fig. 2. If it is assumed that the values of $k_{A F}$ and $k_{A N}$ are similar to the radiative and nonradiative rate constants for CBI-alanine and that $k_{B N}$ is independent of the solvent properties, then only $k_{A B}$ and $k_{B A}$ are affected by changes in the polarity of the solvent. In more polar solvents, the equilibrium $\left(K_{e q}=k_{A B} / k_{B A}\right)$ will favor the $\mathrm{CT}$ state of $\mathrm{CBI}_{2}$-lysine with respect to the $\mathrm{LE}$ state. The results given in Fig. 3 are consistent with these conclusions. The slope of the plot in Fig. $3(0.125)$ reflects the sensitivity of the LE/CT equilibrium towards solvent polarity. The equilibrium, as already stated, shifts in favor of the CT state as the solvent polarity is increased. The present results do not allow us to assign values to $k_{A B}$ and $k_{B A}$. However, it may be that the fluorescence decay is dominated by $k_{A B}$. If so, the expression: ${ }^{16,17}$

$$
k=k^{\prime} \exp \left[A\left(E_{t}(30)-30\right)\right] / R T
$$

is appropriate, where $k^{\prime}$ is the polarity-corrected rate constant and $A$ is the sensitivity of the barrier height to solvent polarity (the slope of Fig. 3). It should be noted, however, that the rate constant $k_{B A}$ may not be negligible when compared to $k_{A B}$ in the solvent systems investigated. If it were, the ratio $\phi_{f} / \tau_{f}$ should be independent of any changes in the solvent polarity. Inspection of the ratio $\phi_{f} / \tau_{f}$ for $\mathrm{CBI}_{2}$-lysine (see Tables II and III) indicates that this ratio is not constant, but actually increases as the polarity of the solvent is increased. Furthermore, the establishment of a rapid pre-equilibrium between the LE and CT states cannot be assumed. If equilibrium populations of these states were established, it can be shown that the ratio $\phi_{f} / \tau_{f}$ would be equal to the quantity $k_{r} /\left(K_{e q}+1\right)$. As the solvent polarity is increased, $K_{e q}$ is expected to increase, and $\phi_{f} / \tau_{f}$ would decrease, contrary to our results (Tables II and III).

The apparent negative activation energy seen from the data of Table IV and shown in the plot of Fig. 4 is similar to the results obtained for the DMABN system reported by Eisenthal and co-workers. ${ }^{16,17}$ The barrier height between the LE and CT states is effectively decreased at lower temperatures (negative activation energy) because of the increased polarity of the solvents at lower temperatures. The slope of a plot of $\ln k^{\prime}$ (the polaritycorrected rate constant obtained from Eq. 5) versus 1/T should yield the activation energy between the LE and CT states of $\mathrm{CBI}_{2}$-lysine. Such a plot for the data of Fig. 4 yields a polarity-corrected activation energy of $3.2 \mathrm{kcal} /$ mole, consistent with $\mathrm{C}$ - $\mathrm{C}$ single-bond rotational energies. However, the assumption underlying the validity of Eq. 5 is that $k_{A B} \gg k_{B A}$. As discussed earlier, $k_{B A}$ may not be negligible and, therefore, the calculation of the activation energy between the LE and CT states through the use of Eq. 5 should be regarded as provisional. In
TABLE $V$. The fluorescence quantum efficiencies $\left(\phi_{f}\right)$ and lifetimes $\left(\tau_{f}\right)$ for CBI-alanine and $\mathrm{CBI}_{2}$-lysine in glycerol/water binary solvent mixtures. The radiative $\left(k_{v}\right)$ and nonradiative $\left(k_{n}\right)$ for CBI-alanine are also presented.a

\begin{tabular}{ccccc}
\hline $\begin{array}{c}\text { CBI-alanine } \\
\% \text { Glycerol }^{\mathrm{a}}\end{array}$ & $\tau_{f}(\mathrm{~ns})$ & $\phi_{f}$ & $k_{r}\left(\mathrm{~ns}^{-1}\right)$ & $k_{n r}(\mathrm{~ns})$ \\
\hline $88[78]$ & 19.5 & 0.67 & 0.034 & 0.017 \\
& $( \pm 0.9)$ & $( \pm 0.03)$ & & \\
$92[156]$ & 19.3 & 0.69 & 0.036 & 0.016 \\
& $( \pm 0.9)$ & $( \pm 0.04)$ & & \\
$94[221]$ & 19.8 & 0.66 & 0.033 & 0.017 \\
& $( \pm 0.7)$ & $( \pm 0.04)$ & & \\
$96[359]$ & 19.8 & 0.65 & 0.032 & 0.018 \\
$98[546]$ & $( \pm 0.7)$ & $( \pm 0.05)$ & & \\
& 19.8 & 0.64 & 0.032 & 0.018 \\
\hline
\end{tabular}

\begin{tabular}{ccc}
$\begin{array}{c}\mathrm{CBI}_{2} \text {-lysine } \\
\% \text { Glycerol }\end{array}$ & $\tau_{f}(\mathrm{~ns})$ & $\phi_{f}$ \\
\hline $88[78]$ & 12.9 & 0.12 \\
& $( \pm 0.6)$ & $( \pm 0.02)$ \\
$92[156]$ & 13.3 & 0.17 \\
& $( \pm 0.6)$ & $( \pm 0.02)$ \\
$94[221]$ & 15.4 & 0.19 \\
& $( \pm 0.5)$ & $( \pm 0.02)$ \\
$96[359]$ & 16.1 & 0.20 \\
$98[546]$ & $( \pm 0.5)$ & $( \pm 0.03)$ \\
& 16.6 & 0.23 \\
& $( \pm 0.7)$ & $( \pm 0.03)$ \\
\hline
\end{tabular}

a The numbers given in brackets are the viscosities of the binary solvent mixture.

b The numbers in parentheses represent the standard deviations.

order to clarify these issues, a direct measurement of $k_{A B}$ is needed.

The effect of solvent viscosity on the fluorescence property of $\mathrm{CBI}_{2}$-lysine indicates that a molecular conformational change is associated with the formation of the CT state. The orientation of the isoindole rings in the ground state of $\mathrm{CBI}_{2}$-lysine, when adsorbed on a solid substrate, does not result in efficient CT state formation, because the fluorescence emission of $\mathrm{CBI}_{2}$-lysine is very close to that of CBI-alanine. Similarly, a high-viscosity solution also impedes $\mathrm{CT}$ state formation. A possible reason is that the "sandwich" conformation, in which the isoindole rings are essentially parallel to each other in close proximity, is unlikely to be attained during the lifetime of the LE excited state. This assumes that a molecular conformational change is required during the excited state for CT formation.

Large overall conformational changes necessary to achieve the structure required for CT state formation may seem unlikely during the excited-state lifetime because of entropic effects and the relatively large size of the isoindole rings. Nevertheless, the reduced quantum yields for $\mathrm{CBI}_{2}$-lysine in low-viscosity solvents are consistent with an efficient CT state formation.

A computer graphical search was conducted to assess whether any particular ground-state conformation was preferred for $\mathrm{CBI}_{2}$-lysine. All C-C and C-N single bonds were allowed to freely rotate, and the total energy associated with each conformation was the sum of the energy due to torsional strain, nonbonded interactions, bond stretching, bond bending, electrostatics, and van der 


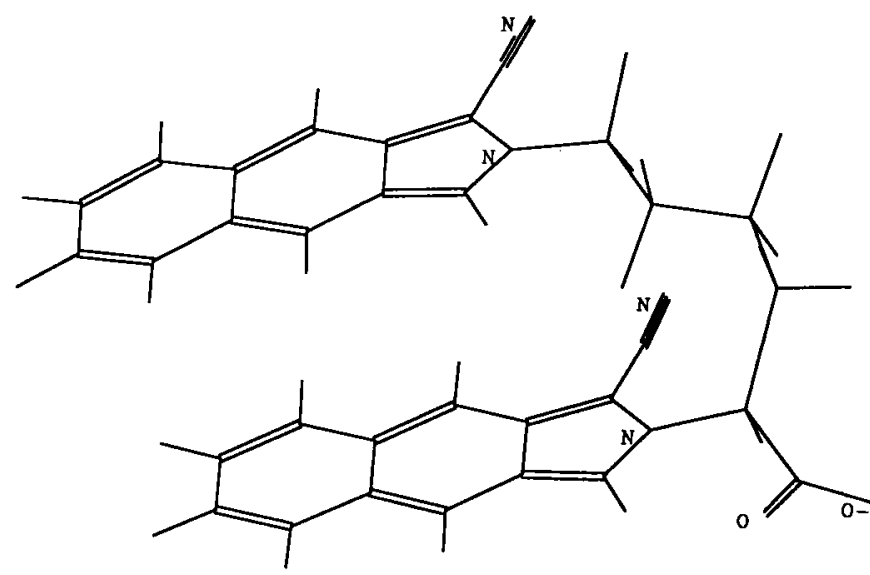

Fig. 5. A low-energy molecular conformation of $\mathrm{CBI}_{2}$-lysine obtained from the computer ground-state conformational search.

Waals interactions. No solvent or $\pi-\pi$ interactions were considered (they could be repulsive in nature). Several low-energy conformers were found, one of which is shown in Fig. 5. As is evident from this conformation, the isoindole rings may be in a stacked, sandwich-type configuration that places the rings in close proximity to one another. Although no solvent interactions were included in the computational search, a stacked-type geometry would be favored in polar solvents because of the minimization of any hydrophobic interactions between the solvent and the isoindole rings. The distance between the isoindole rings in the conformer is $\sim 4 \AA$, thereby precluding solvent from being between the two isoindole rings. The number of $\mathrm{C}-\mathrm{C}$ or $\mathrm{C}-\mathrm{N}$ bond rotations required to attain such a sandwich-type conformation in this case would be minimal in comparison to a conformation in which the isoindole rings are far apart and unstacked. The energy associated with this latter type of conformer results in the predicted energy being considerably higher in comparison to the conformer shown in Fig. 5. Qualitatively, the ground-state conformation favored by the computational modeling is consistent with the observed fluorescence properties of $\mathrm{CBI}_{2}$-lysine. The effects of viscosity on the observed $\phi_{f}$ and $\tau_{f}$ of $\mathrm{CBI}_{2}$-lysine indicate that the required conformation for the CT state is not identical to that of the ground state. Further investigations are required in order to determine the degree of reorientation required for $\mathrm{CT}$ formation.

The absence of a fluorescence band red-shifted from the fluorescence band of the LE state indicates that the fluorescence quantum efficiency of the CT state is extremely small in the solvent systems investigated. Although the CT state is stabilized by more polar solvents, the nonradiative processes for depopulation of the CT state overwhelm the radiative process and thus result in reduced fluorescence yields. This observation has been seen in a number of different systems. ${ }^{21-24}$ The apparent lack of fluorescence from the $\mathrm{CT}$ state of $\mathrm{CBI}_{2}$-lysine precludes fluorescence methods as a means to obtain direct kinetic measurements on this state. An alternative method would be the use of time-resolved absorption spectroscopy ${ }^{25-29}$ which could provide information on the electronic structure and the decay dynamics of that excited state.

\section{ACKNOWLEDGMENTS}

The authors would like to express their gratitude to Oread Laboratories, the Kansas Technology Enterprise Corporation, and the $\mathrm{Na}$ tional Science Foundation (S.C., REU program) for financial support of this work. The authors would also like to thank Dr. Ken Audus, for his help is obtaining the lifetime measurements, and Kit Gunn, for his assistance in performing the computer molecular modeling. S.A.S. would also like to thank the Pittsburgh Conference for a Summer Fellowship Award in Analytical Chemistry, administered by the American Chemical Society.

1. R. Carlson, K. Srinivasachar, R. Givens, and B. Matuszewski, J. Org. Chem. 51, 3978 (1987).

2. B. Matuszewski, R. Givens, K. Srinivasachar, R. Carlson, and T. Higuchi. Anal. Chem. 59, 1102 (1987).

3. P. DeMontigny, J. Stobaugh, R. Givens, R. Carlson, L. Sternson, K. Srinivasachar, and T. Higuchi, Anal. Chem. 59, 1096 (1987).

4. P. Montigny, Ph.D. thesis, the University of Kansas, Lawrence (1986).

5. M. Roach and M. Harmony, Anal. Chem. 59, 411 (1987).

6. B. Nickerson and J. Jorgenson, J. of High Resol. Chromatogr. and Chromatogr. Comm. 10744, 533 (1989).

7. S. Soper, S. Lunte, and T. Kuwana, Anal. Sci. 5, 23 (1989).

8. Fjalar Kristjansson, Ph.D. thesis, the University of Kansas, Lawrence (1987)

9. S. Soper and T. Kuwana, Appl. Spectrosc. 43, 883 (1989).

10. E. Chandross and H. Thomas, Chem. Phys. Lett. 9, 393 (1971).

11. F. Hirayama, J. Chem. Phys. 42, 3163 (1965).

12. J. Birks, Nature 214, 1187 (1967).

13. E. Chandross and C. Dempster, J. Am. Chem. Soc. 92, 3586 (1970).

14. A. Amiri, Chem. Phys. 125, 145 (1988).

15. E. Lippert, W. Luder, and H. Boss, in Advances In Molecular Spectroscopy (Pergamon, Oxford, 1962), p. 443.

16. J. Hicks, M. Vandersall, E. Sitzmann, and K. Eisenthal, Chem. Phys. Lett. 116, 18 (1985).

17. J. Hicks, M. Vandersall, E. Sitzmann, and K. Eisenthal, Chem. Phys. Lett. 135, 413 (1987).

18. K. Al-Hassan and T. Azumi, Chem. Phys. Lett. 146, 121 (1988).

19. S. Strikler and R. Berg, J. Chem. Phys. 37, 814 (1962).

20. C. Reichardt, in Molecular Interaction (John Wiley and Sons, New York, 1982), p. 241.

21. H. Knibbe, K. Rollig, F. Schafer, and A. Weller, J. Chem. Phys. 47, 1184 (1967).

22. N. Mataga, T. Okada, and N. Yamamoto, Chem. Phys. Lett. 1, 119 (1967).

23. T. Okada, T. Fujita, M. Kubota, S. Masaki, and N. Mataga, Chem. Phys. Lett. 14, 563 (1972).

24. G. Beddard and R. Davidson, J. Photochem. 1, 491 (1972).

25. Y. Taniguchi, Y. Nishina, and N. Mataga, Bull. Chem. Soc. Japan 45, 764 (1971).

26. N. Mataga, Pure \& Appl. Chem. 56, 1255 (1984).

27. N. Mataga, H. Shioyama, and Y. Kanda, J. Phys. Chem. 91, 314 (1987).

28. C. Rulliere, Z. Grabowski, and J. Doblowski, Chem. Phy. Lett. 137, 408 (1987)

29. N. Mataga, H. Yao, T. Okada, and W. Rettig, J. Phys. Chem. 93, 3383 (1989). 